\title{
LIDIANDO CON LA FRONTERA VERTICAL: ESTRATEGIAS MIGRATORIAS DE LOS HONDUREÑOS EN TRÁNSITO POR MÉXICO
}

\author{
Dealing with the vertical border: migration strategies \\ of Hondurans in transit through Mexico
}

José Carlos Yee Quintero ${ }^{1}$

Eduardo Torre Cantalapiedra²

\begin{abstract}
Resumen. Este trabajo analiza las estrategias implementadas por los migrantes hondureños en tránsito por México ante los puntos de control desplegados por las autoridades mexicanas y la presencia de criminales dedicados a la explotación de migrantes. Estas vicisitudes al interior del territorio, denominadas 'la frontera vertical', llevan a los migrantes a reconfigurar constantemente su ejercicio de tránsito. Para analizar dichas estrategias y la forma en que se presentan se realizó trabajo de campo en Saltillo, Coahuila, donde se aplicaron entrevistas semiestructuradas. En otro acercamiento, para comprender la composición de la frontera vertical mexicana se utilizaron registros administrativos de la SEGOB y de la Encuesta sobre Agresiones y Abuso a Migrantes ${ }^{3}$.
\end{abstract}

Palabras clave: migración internacional, políticas inmigratorias mexicana, hondureños, migración en tránsito, frontera vertical.

Abstract. This paper analyzes the strategies implemented by the Honduran migrants in transit through Mexico to deal with the checkpoints deployed by Mexican authorities and the presence of criminals dedicated to the exploitation of migrants. These vicissitudes within the territory, called 'vertical border', lead migrants to constantly reconfigure their migratory exercise. To analyze these strategies, and how they appear, fieldwork was conducted in Saltillo, Coahuila, where

1 El Colegio de la Frontera Norte. Tijuana, México.

2 UNAM. Programa de Becas Posdoctorales en la UNAM, Becario del Instituto de Investigaciones Jurídicas. Ciudad de México, México.

3 Este trabajo recibió financiamiento del Fondo Conacyt Investigación Científica Básica 2012, Proyecto 0178078, Flujos migratorios en las fronteras norte y sur de México y en tránsito hacia Estados Unidos: dinámicas poblacionales y políticas migratorias, coordinado por María Eugenia Anguiano Téllez, El Colegio de la Frontera Norte. 
semi-structured interviews were applied. In another approach, to understand the composition of the Mexican vertical border, SECOB administrative records and data from the survey about Assault and Abuse to Migrants, were used.

Keywords: international migration, Mexican immigration politics, Hondurans, transit migration, vertical border.

\section{Introducción ${ }^{4}$}

En las últimas décadas, las políticas de control del flujo migratorio centroamericano que carece de documentos, -en particular, el de hondureños-, se han endurecido en México; un país que se ha consolidado como de tránsito de migrantes ${ }^{5}$. A partir del año 2014, el Programa Frontera Sur ha añadido nuevas dificultades para el tránsito de migrantes indocumentados por México.

En este sentido toma importancia el análisis de una frontera que se reconstruye a lo largo de México y de forma paralela a las principales rutas migratorias con el fin de obstaculizar el flujo de centroamericanos y evitar su llegada a Estados Unidos (EUA). Los migrantes en tránsito por México -en su mayoría de origen centroamericano- tienen una exposición constante al riesgo de deportación durante todo su trayecto. Además, esto conlleva otro tipo de prácticas inherentes a la presencia de migrantes y agentes del Estado ${ }^{6}$ relacionada con estructuras de violencia y explotación de los migrantes. Aunado a lo anterior, existe un conjunto de agentes no estatales que perpetran abusos y agresiones en contra de los migrantes.

En este contexto, el desarrollo de estrategias para lidiar con estos obstáculos y riesgos del camino por parte de los migrantes resulta trascendental. Las estrategias de estos frente a un entorno de políticas en su contra es un campo de estudio que ha sido explorado por trabajos recientes desde una óptica de la conformación transnacional de familias ${ }^{7}$; y desde perspectivas como la adaptación de familias a las políticas anti-inmigrantes ${ }^{8}$. En ambos casos

4 Agradecemos el apoyo de la Casa del Migrante de Saltillo y su equipo de trabajo, quienes hicieron posible el trabajo de campo para esta investigación. Además agradecemos los comentarios de Brígida García a una versión anterior de este trabajo.

5 CASTILLO, Manuel Ángel. Las políticas hacia la migración centroamericana en países de origen, de destino y de tránsito.

6 En este artículo, se entiende por "agente del Estado" a cualquier representante del Estado mexicano: miembros de alguna corporación policiaca, agentes y funcionarios del Instituto Nacional de Migración, ejército o la marina. Asimismo, se entiende por "agente no estatal" a otros actores que dificultan el tránsito migratorio tales como criminales y crimen organizado.

7 PEDONE, Claudia, ECHEVERRI, María Margarita, GIL ARAUJO, Sandra. Entre dos orillas. Cambios en las formas de organización de las familias migrantes latinoamericanas en España en tiempos de crisis global.

8 TORRE CANTALAPIEDRA, Eduardo, ANGUIANO TÉLLEZ, María Eugenia. Viviendo en las sombras: estrategias de adaptación de familias inmigrantes mexicanas en Arizona, 2007-2015. 
las estrategias conducen a dar, o a devolver, el protagonismo a los propios migrantes.

La Secretaría de Gobernación reportó que en 2014 los hondureños representaron el $38.6 \%$ de los extranjeros devueltos por México, aunado a sus dimensiones, se les identifica en la literatura como una población expuesta a situaciones que impactan en su capacidad para generar estrategias, por ejemplo: tener escasas redes de apoyo y experiencia migratoria generacional por ser un flujo reciente; así como por sus condiciones de salida adversas a la luz de la violencia en Honduras.

Nuestro objetivo es identificar y analizar las estrategias que generan los migrantes hondureños para superar los obstáculos de la frontera vertical. Para lograrlo, el documento está dividido en tres partes: 1) los hondureños frente a la frontera vertical, donde utilizando datos administrativos y de la Encuesta sobre Agresión y Abuso a Migrantes (EAAM) 2012 se ilustra la existencia de una frontera vertical y el contacto con ella de la población hondureña; 2) estrategias de los migrantes en tránsito, donde se desarrolla la vinculación entre la frontera vertical y las estrategias analizadas, las cuales serán entendidas como una expresión de la agencia del migrante ${ }^{9}$; y 3) las estrategias de los hondureños en tránsito, donde con base en 24 entrevistas semiestructuradas realizadas a migrantes en Saltillo, Coahuila, se recuperan y analizan tres tipos de estrategias: i) que involucran la elección de rutas y medios de transporte, ii) que involucran el acompañamiento y iii) que involucran acciones concretas ante peligros en el tránsito (agentes del Estado y criminales); teniéndose en cuenta tres ejes de análisis transversal: el género, la información de la que disponen y los recursos económicos que poseen. Finalmente se presentan un conjunto de reflexiones finales a modo de conclusión.

\section{Los migrantes hondureños en tránsito por México frente a la frontera vertical}

Los migrantes centroamericanos indocumentados, que atraviesan el territorio mexicano con destino a EUA, hacen frente a una serie de medidas de control vinculadas a la deportación que ha sido denominada "frontera vertical"10. Esto evidencia que las características asociadas a los espacios fronterizos, en lo que respecta al control migratorio, se extienden ahora hacia el

\footnotetext{
9 SILVA HERNÁNDEZ, Aída. Estrategias de tránsito de adolescentes centroamericanos independientes: enfrentando la frontera vertical en México.

${ }^{10}$ Véanse, por ejemplo, GONZÁLEZ VELÁZQUEZ, Eduardo. Frontera vertical: México frente a los migrantes centroamericanos; TORRE-CANTALAPIEDRA, Eduardo, SCHIAVON, Jorge A. Actuar o no actuar: un análisis comparativo del rol de los estados de Chiapas y Arizona en la gestión de la inmigración.
} 
interior del país ${ }^{11}$. Asimismo, una "frontera vertical extraoficial" se "...superpone a la primera, obstaculizando el tránsito de manera tácita a través de grupos delictivos que se sustentan en la explotación de la persona inmigrante, no sólo en términos económicos y laborales, sino corporales"12. La línea divisoria entre ambas fronteras no siempre es clara, puesto que en ocasiones los agentes del Estado están coludidos con grupos criminales.

A continuación, para entender bajo qué condiciones se produce el tránsito de los migrantes hondureños y centroamericanos por México, se muestran y analizan las rutas que éstos recorren y los obstáculos a los que se enfrentan. Antes de proceder con estos análisis es adecuado dar cuenta de la gran vulnerabilidad de la población hondureña en tránsito. Casillas ha encontrado que la población centroamericana indocumentada es la más vulnerable y que dentro de éstos "quienes con más frecuencia reúnen estas condiciones y circunstancias [que los hacen vulnerables a agresiones y abusos] son los nacionales de Honduras"13.

Su perfil vulnerable se puede entender mejor si se da cuenta de las condiciones que originaron su emigración. Honduras sufrió en 1998 el Huracán Mitch, que tuvo consecuencias devastadoras en términos de vidas humanas, pero también en términos de destrucción de las condiciones de vida generales, lo que supuso la generación de una importante oleada migratoria hacia EUA. Los flujos fueron creciendo hasta llegar a su culmen en el año 2005. En cualquier caso los flujos siguen siendo especialmente importantes una década después, puesto que existen otros factores importantes de expulsión, como son la creciente violencia y las condiciones de pobreza y desigualdad en Honduras.

Son varias las principales rutas terrestres seguidas por los centroamericanos en tránsito por México, tanto en el norte como en el sur ${ }^{14}$. Los datos administrativos sobre devoluciones apuntan a que los migrantes hondureños en el norte tienden a ir por las rutas más hacia el este del país (Cuadro 1), las cuales minimizan la distancia recorrida por México para llegar a EUA, pero que son consideradas las más peligrosas. Así, las entidades federativas de Tamaulipas, Coahuila y Nuevo León son las tres que presentan, con diferencia, un mayor número de migrantes capturados por las autoridades mexicanas. Siendo una ruta relevante la que atraviesa por la ciudad de México y llega a Saltillo (Coahuila), sitio donde se realizaron las entrevistas a migrantes.

\footnotetext{
11 ANGUIANO TÉLLEZ, María Eugenia, TREJO PEÑA, Alma Paola. Vigilancia y control en la frontera México-Estados Unidos: efectos en las rutas del flujo migratorio internacional.

12 SILVA HERNÁNDEZ, op. cit., p. 100.

${ }^{13}$ CASILLAS, Rodolfo. Los migrantes indocumentados: su vulnerabilidad y la nuestra, p. 149.

${ }^{14}$ Véase al respecto IDEM. Una vida discreta, fugaz y anónima: los centroamericanos transmigrantes en México.
} 


\section{Cuadro 1 - Eventos de devolución de migrantes hondureños por la autoridad mexicana, por entidad federativa de la región fronteriza con Estados Unidos, ene./nov. 2015}

\begin{tabular}{|l|c|}
\hline ENTIDAD FEDERATIVA & EVENTOS \\
\hline Tamaulipas & 1,076 \\
\hline Coahuila & 862 \\
\hline Nuevo León & 412 \\
\hline Sonora & 254 \\
\hline Baja California & 175 \\
\hline Chihuahua & 117 \\
\hline
\end{tabular}

Fuente: Elaboración propia con base en las estadísticas del INM, 2015.

En la gráfica 1, aparece la evolución en el volumen de devueltos a lo largo de los últimos años (2007-2015), y se puede observar como la tendencia a la caída en volumen, es de aproximadamente 60 mil detenciones en el año 2011, le sucede una evolución alcista siendo las devoluciones en el año 2015 más del doble que cuatro años atrás (llegando casi a los 145 mil, entre enero y noviembre). Hay que tener en cuenta que las variaciones en el número de eventos no sólo corresponden a las variaciones en los flujos de migrantes en tránsito, sino a las producidas en las políticas de control migratorio. En los últimos años, destaca el Programa Frontera Sur que ha cohesionado diversas estrategias vinculadas al control de la migración centroamericana en tránsito; y ha contado con el apoyo de EUA con equipamiento y entrenamiento relacionado a seguridad fronteriza, como parte de un paquete de asistencia que ascendía a 2,500 millones de dólares ${ }^{15}$.

En este sentido los datos referentes a las devoluciones de migrantes por México apuntan efectivamente a que se ha instaurado una frontera vertical. En la gráfica 1 puede observarse que cada montante anual está dividido en función de cuatro regiones -frontera sur, sur, centro norte ${ }^{16}$, , conformadas de acuerdo a una lógica de la distancia recorrida desde el cruce de la línea fronteriza. Donde se observa que consistentemente un importante número de devoluciones (aproximadamente la mitad en el año 2015) no se realizan en ninguno de los estados fronterizos ${ }^{17}$. Otra consideración es el aumento a partir de 2014, año en que inicio el Plan Frontera sur, de las capturas en la región centro y sur.

\footnotetext{
${ }_{15}$ PROJECT COUNSELLING SERVICE. El programa frontera sur y la política migratoria en la franja.

${ }^{16}$ Las entidades federativas que componen cada una de las cuatro regiones son: frontera sur (Campeche, Chiapas, Quintana Roo y Tabasco), sur (Oaxaca, Veracruz y Yucatán), centro (Aguascalientes, Colima, Ciudad de México, Guanajuato, Guerrero, Hidalgo, Jalisco, México, Michoacán, Morelos, Nayarit, Puebla, Querétaro, San Luis de Potosí, Tlaxcala y Zacatecas) y norte (Baja California, Baja California Sur, Coahuila, Chihuahua, Durango, Nuevo León, Sinaloa, Sonora y Tamaulipas).

17 Aunque no se presentan, se realizaron cálculos para dar cuenta que la distribución por regiones de los eventos de devolución de los migrantes hondureños era muy similar a las del resto de la población.
} 


\section{Gráfica 1 - Distribución por regiones de eventos de devolución de migrantes por la autoridad mexicana, 2007-2015}

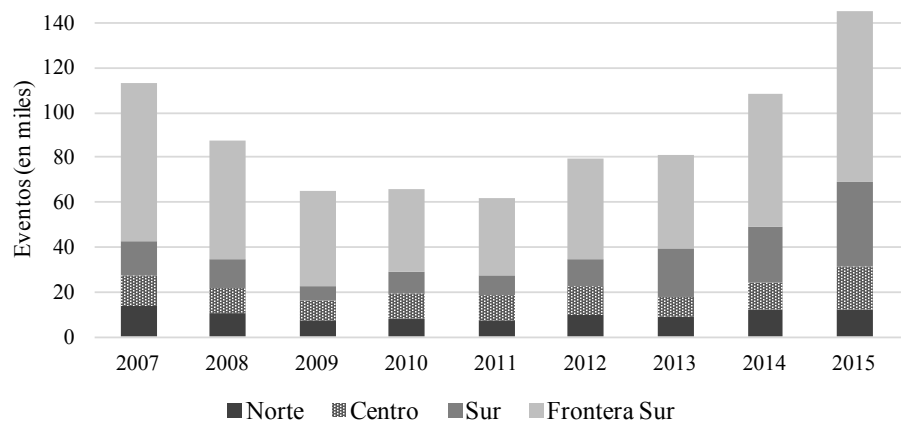

Nota: La columna del año 2015 sólo refleja las devoluciones acaecidas entre enero y noviembre. Fuente: Elaboración propia con base en las estadísticas del INM, 2007-2015.

La gráfica 2 muestra que los países centroamericanos son quienes concentran la mayor parte del volumen de devoluciones conducidas por las autoridades mexicanas $y$, por tanto, las que sufren el sistema de control migratorio vertical implementado por México. La cifra de hondureños aparece en un segundo lugar durante el periodo analizado, por debajo de la población guatemalteca, lo cual podría estar impactado como sugiere Fernánde $z^{18}$ por mecánicas migratorias diferenciadas entre los centroamericanos. En particular por prácticas de la población guatemalteca que tiene una gran presencia periódica en el sur de México, sin constituir migración de tránsito puesto que sólo cruzan a trabajar diaria, semanal o mensualmente, lo que no impide sean objeto de detención y devolución por parte de las autoridades del INM.

\section{Gráfica 2 - Distribución de los eventos de devolución de migrantes por la autoridad mexicana por país de procedencia, 2007-2015}

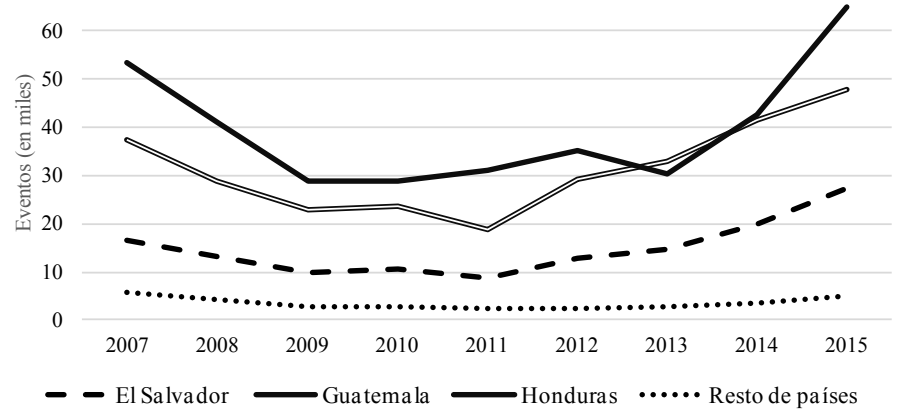

Nota: La columna del año 2015 sólo refleja las devoluciones acaecidas entre enero y noviembre. Fuente: Elaboración propia con base en las estadísticas del INM, 2007-2015.

${ }_{18}$ FERNÁNDEZ CASANUEVA, Carmen. Movilidad y asentamiento: estrategias de migrantes y trabajadores transfronterizos para vivir y laborar en la región de El Soconusco, Chiapas. 
La literatura ha señalado que la población migrante hondureña es la más propensa a ser violentada en México ${ }^{19}$. "Las y los migrantes de nacionalidad hondureña fueron quienes mayores casos de delitos cometidos en su contra registraron (52.89\%) [Datos de la REDODEM]"20. Asimismo, Calva et alii utilizando la EAAM subrayan que la incidencia de agresiones y abusos a la población hondureña es la mayor entre los flujos de centroamericanos $(15.7 \%)^{21}$.

Utilizando esa misma encuesta, EAAM, se realizó el cuadro 2 que muestra la distribución de las agresiones y abusos sufridos por los migrantes hondureños, durante el año previo a la fecha de la entrevista entre los devueltos por las autoridades de EUA. Se clasifican estás de acuerdo a las cuatro regiones ya anteriormente empleadas -norte, centro, sur y frontera sur- lo que ayuda a dimensionar la frontera vertical extraoficial vinculada con criminales que explotan migrantes. Si bien el cuadro 2 no recoge toda la violencia contra los migrantes hondureños en tránsito, aporta evidencia de que las agresiones y abusos se dan a lo largo de toda su ruta por México. Asimismo, se puede observar de forma diferenciada las agresiones y abusos a migrantes hondureños por agentes del Estado y por criminales comunes o miembros del crimen organizado.

\section{Cuadro 2 - Migrantes hondureños devueltos por autoridades de EUA: distribución de agresiones y abusos por regiones, durante un año previo a la fecha de la entrevista, por tipo de agresor}

\begin{tabular}{|l|l|c|}
\hline \multirow{4}{*}{ Norte } & & Hondureños \\
\hline \multirow{4}{*}{ Centro } & Agente del Estado & 88 \\
\cline { 2 - 3 } & Criminales y Crimen Organizado & 188 \\
\cline { 2 - 3 } & Total & 276 \\
\hline \multirow{4}{*}{ Sur } & Agente del Estado & 28 \\
\cline { 2 - 3 } & Criminales y Crimen Organizado & 260 \\
\cline { 2 - 3 } & Total & 288 \\
\hline \multirow{4}{*}{ Frontera sur } & Agente del Estado & 78 \\
\cline { 2 - 3 } & Criminales y Crimen Organizado & 140 \\
\cline { 2 - 3 } & Total & 218 \\
\cline { 2 - 3 } & Criminales y Crimen Organizado & 75 \\
\cline { 2 - 3 } & Total & 433 \\
\hline
\end{tabular}

Fuente: Elaboración propia con base en la Encuesta sobre Agresión y Abuso a Migrantes (oct. 2011- sep. 2012).

\footnotetext{
${ }^{19}$ YEE QUINTERO, José Carlos. Las víctimas del Estado: Violencia hacia migrantes hondureños, perpetrada por agentes del Estados, durante su tránsito por México.

${ }^{20}$ REDODEM. Migrantes Invisibles, violencia tangible. Informe 2014, p. 67.

${ }^{21}$ CALVA, Luis et alii. Principales Resultados de la Encuesta Sobre Agresión y Abuso a Migrantes (EAAM) devueltos por las autoridades migratorias, 2012.
} 
En síntesis, son diversas las vicisitudes que los migrantes hondureños deben enfrentar en su tránsito por México, dado que tanto las deportaciones como los abusos y agresiones por agentes estatales y no estatales se extienden por todo el territorio nacional. Lo que lleva a centrar los cuestionamientos a aspectos puntuales, por ejemplo: a pesar de la escasez de recursos con los que cuentan, ¿̇son los migrantes hondureños capaces de generar estrategias con la finalidad de hacer más probable el éxito de su tránsito por México?

\section{Estrategias de los migrantes en tránsito}

En este trabajo, se cuestiona si los migrantes están dotados de una cierta capacidad de agencia para lidiar con las vicisitudes interpuestas por una frontera vertical de doble cariz -oficial y extraoficial-, mediante el desarrollo de determinadas estrategias. Para lo cual en este apartado se desarrollan los elementos teórico-conceptuales requeridos para el análisis de la información cualitativa. Se parte de Rivas, quien tras una discusión exhaustiva sobre la agencia en relación a los migrantes centroamericanos en tránsito por México, retoma las propuestas en Long'22:

...la propuesta de Long inclinaría la balanza sobre la agencia humana en la ecuación estructura/actor, en contraposición a los análisis macrosociales e institucionales, que priman los constreñimientos estructurales -una política migratorio restrictiva, por ejemplo- sobre la acción de los individuos. (...) más que de agentes hay que hablar de actores dando la impresión de que concede más libertad a los individuos que en los postulados de Sewell, Giddens y Bourdieu... ${ }^{23}$.

Si bien no se reconstruirá la exploración teórica respecto a la agencia hasta un nivel tan profundo al realizado por estos autores, es importante destacar la forma en que para el caso de los migrantes y específicamente de los centroamericanos toma fuerza un enfoque que reconoce las acciones del migrante como algo central para comprender las estrategias migratorias. Como lo establece Long "una ventaja del enfoque centrado en el actor es que se empieza con el interés de explicar las respuestas diferenciales a circunstancias estructurales similares, aun cuando las condiciones parezcan más o menos homogéneas" ${ }^{24}$.

La necesidad de una perspectiva de este tipo se relaciona con un aparato de control migratorio que complejiza cada vez más el tránsito por México, aunado con lo que Rivas observa del migrante como un individuo que no es en extremo racionalmente calculador, pero tampoco cuenta con libertad absoluta. En este sentido sitúa al migrante en un punto intermedio donde cuenta con una agencia observable, nutrida por aspectos como su experiencia.

\footnotetext{
${ }^{22}$ LONG, Norman. Sociología del desarrollo: Una perspectiva centrada en el actor.

${ }^{23}$ RIVAS CASTILLO, Jaime. ¿Víctimas nada más?: migrantes centroamericanos en el Soconusco, Chiapas.

${ }^{24}$ LONG, op. cit., p. 43.
} 
...los migrantes son el objetivo de políticas migratorias restrictivas y a veces francamente represivas, mediante la implementación de operativos que incrementan el riesgo de sufrir un aseguramiento violento e ilegal, por ejemplo: ante ello, los migrantes han desarrollado una serie de estrategias orientadas a evadir los controles o enfrentarse a ellos en caso de un encuentro indeseado. Adscribirse a una red social de apoyo o construir una son ya estrategias ${ }^{25}$.

De acuerdo a lo anterior, se considera que el uso de un concepto de estrategias desarrollado a partir del de Silva ${ }^{26}$, resulta idóneo para este trabajo. Así se entiende que las estrategias migratorias en tránsito se constituyen por el uso por parte de los migrantes en tránsito, entendidos como agentes, de los recursos personales, interpersonales e institucionales que es capaz de poner a su alcance, en una secuencia de decisiones y acciones que se dirigen a hacer frente a los obstáculos que se interponen a la consecución de su tránsito migratorio.

En seguimiento al concepto que se utilizará de estrategias migratorias es posible observar que éstas se materializan específicamente en decisiones y acciones para soslayar los obstáculos migratorios. Al margen de esto pareció adecuado retomar la perspectiva planteada en Ruiz, quien define el riesgo migratorio como un proceso con diversos componentes o situaciones ${ }^{27}$. Esta autora construye una tipología de factores que impactan el riesgo de la población centroamericana en tránsito, los cuales se retoman como base para la agrupación de los principales tipos de estrategias exploradas en este trabajo. Esto de acuerdo a la esencia de que una estrategia se despliega gracias a la agencia que posee el migrante ante un riesgo potencial o ante un encuentro directo con un riesgo. Los tres tipos de factores desarrollados por Ruiz son: los migrantes; peligros potenciales u objetos de riesgo; y el encuentro o contacto.

El primer factor, referente a los migrantes, será considerado de forma transversal al análisis, pues sus características se encuentran presentes a lo largo de la conformación de las diversas estrategias. Lo contenido en él se vincula, desde otras perspectivas de análisis, con la vulnerabilidad abordada en París donde se observa a las diferencias demográficas como un grupo de factores que pueden impactar en la vulnerabilidad del migrante en tránsito ${ }^{28}$. En este sentido se delimitaron tres ejes transversales a analizar que están presentes en las estrategias de los migrantes en tránsito: el género, la información con la que cuentan y los recursos económicos que poseen.

\footnotetext{
${ }^{25}$ RIVAS CASTILLO, op. cit., p. 29-30.

${ }^{26}$ SILVA HERNÁNDEZ, op. cit.

${ }^{27}$ RUIZ, Olivia. La Migración Centroamericana en la Frontera Sur: un Perfil del Riesgo en la Migración Indocumentada Internacional.

${ }^{28}$ PARÍS, María Dolores. Vulnerabilidad de jóvenes migrantes en el cruce indocumentado de la frontera México-Estados Unidos. Procesos mexicanos y centroamericanos.
} 
El segundo tipo de factores de Ruiz, peligros potenciales u objetos de riesgo, se conforma por "...personas que actúan con la intención de hacer algo que puede causar (muy probablemente causará) un daño (sea físico, psicológico o emocional) en el o la migrante o perjudicar la migración (interrumpir, detener o cancelar el viaje)..."29. En ella se pueden agrupar tanto a los criminales y bandas delictivas como a los agentes del Estado. El aspecto más relevante de este apartado es que se conjugan peligros potenciales no materializados, por lo que las estrategias que se agrupan en él dibujan respuestas a priori de los riesgos de la frontera vertical, es decir que tienen un carácter preventivo. Estas se desarrollarán en dos grupos: estrategias que involucran elección de rutas y medios de transporte; y estrategias que involucran la búsqueda de acompañamiento.

Por último, el tercer factor relacionado con los riesgos hace referencia al encuentro o contacto. Este tiene un carácter muy concreto y tiene lugar cuando se da "...el encuentro o contacto entre el o la migrante y el peligro potencial. En otras palabras, el proceso envuelve un encuentro físico entre la persona y el objeto de riesgo ${ }^{\prime \prime 30}$. De él devendrá un tercer tipo de estrategias relacionadas de forma más directa con la agencia de los migrantes al encontrarse con eventos que pueden detener o perjudicar su ejercicio migratorio: estrategias que involucran acciones ante dificultades directas (agentes del Estado o criminales).

\section{Las estrategias de los hondureños en tránsito}

Para abordar las estrategias migratorias de estos migrantes frente a la frontera vertical se tomó información de 24 entrevistas semiestructuradas ${ }^{31}$ aplicadas en la ciudad de Saltillo, Coahuila. Las entrevistas se aplicaron en enero de 2016 y tomaron en consideración a migrantes que hubieran comenzado su tránsito desde Honduras como máximo 12 meses atrás, lo que les dota de una forma de cohesión. El haber realizado el trabajo de campo en la Casa del migrante de Saltillo (CDMS) permite analizar a una población con una ruta migratoria común, además los directivos de esta casa estiman que alrededor del $80 \%$ de los migrantes que reciben son hondureños.

Debe considerarse que el realizar el trabajo de campo en la CDMS implica que los resultados se avocan a la población que utiliza este tipo de recursos, quienes por lo general tienen condiciones de tránsito particularmente precarias. Aunque no se analiza en este trabajo, acudir a las casas del migrante supone una estrategia en sí mismo de acuerdo a nuestra definición de estrategia de tránsito. A continuación, se abordarán las estrategias según el marco teórico-conceptual construido previamente a través del análisis de las entrevistas. Es importante

\footnotetext{
${ }^{29}$ RUIZ, op. cit., p. 9.

30 Ibidem, p. 10.

${ }^{31}$ Se entrevistaron cuatro a mujeres y veinte a hombres, que en su mayoría viajaban acompañados.
} 
considerar que los diferentes tipos de estrategias, aquí separados por motivos analíticos, se eligieron por su relevancia para el tránsito según la literatura, lo que no las convierte en las únicas ni evita que se entrelacen en la realidad.

\section{Estrategias que involucran la elección de rutas y medios de transporte}

En primer lugar, se identificaron algunas estrategias existentes desde el lugar de origen que impactaron todo el tránsito. En segundo, se procede con las estrategias de elección de rutas y medios de transporte que se desplegaron una vez en tránsito por México. La estrategia de elección de rutas y transporte en el origen es ejemplificada de forma muy clara por Cristian, quien viajó junto con su esposa e hijo de 20 meses con destino a EUA. A pesar de no contar con experiencia migratoria, ellos conocían los riesgos de la migración por lo que decidieron llevar un proceso desde Honduras para obtener una visa de turista mexicana. Por tanto, su estrategia fue perfilada por los recursos económicos con los que contaban y la información sobre el proceso.

Yo conozco muchos que a veces hacen las cosas tal vez por impulso o por desesperación también pero, para todo hay una solución, ziverdad? Solo hay que tener paciencia y tratar de hacer bien las cosas, para nosotros no creas que fue fácil. No fue fácil, nos costó, pero pensando en nuestra hija, en lo mejor por nuestra hija todo nos salió bien, y no tuvo que sufrir todo lo que sufren viniendo en tren... (Cristian).

La estrategia de Cristian y su familia se centró en evitar los riesgos del trayecto por tierra viajando en avión desde Honduras hasta Monterrey. Si bien les tomó más de un año ahorrar lo suficiente para lograrlo, fue posible gracias a una planeación adecuada, en Monterrey contrataron un coyote para que llevara a su esposa e hija a EUA y él se vio obligado a ir a la CDMS, pues no contaban con recursos suficientes para pagar por los tres. La elección de quién debía seguir en solitario parecía obvia: "Ya uno [como hombre] es más diferente, a ver cómo se la juega..." (Cristian).

Como se puede observar, otra estrategia que suele desplegarse desde un inicio es la contratación de traficantes de personas, o coyotes, para toda o una parte del camino. Esto implica rutas y medios de transporte diferentes a los usados por migrantes que viajan por su cuenta, por lo que no es común encontrar en casas del migrante a quienes utilizan coyotes.

A lo largo de los diferentes tramos del tránsito por México, varios de los entrevistados implementaron estrategias preventivas, en cuanto a la elección de medios de transporte al contar con información sobre riesgos. La información cambia durante el tránsito, conforme se gana experiencia migratoria o cuando se está en contacto con otros migrantes, con miembros de casas del migrante 
u otros informantes. Así los migrantes que cuentan con información escogen medios de transporte que no están expuestos a las bandas delictivas, como en el caso de Rogelio, de 47 años y Brandon de 21, quienes viajaban juntos. Rogelio contaba con experiencia migratoria pero no con recursos económicos, por lo que se detenían a trabajar para conseguir dinero en diversos poblados. Ellos consiguieron que un chofer de tráiler les transportara gran parte del viaje, evitando así bandas delictivas, pago de cuotas y demás obstáculos del trayecto migratorio.

En el conjunto de las entrevistas se puede observar, que otra estrategia frecuente consiste en tratar de rodear cualquier obstáculo (por ejemplo, los retenes, zonas controladas por banda criminales, etcétera). Así los migrantes realizan rodeos que involucran cambios de medio de transporte: “...se nos ha complicado mucho porque ahorita no puedes tomar el camión, hay mucha migra" (Pablo). Estas estrategias se presentan en diversos casos, como el de Mario, un migrante sin experiencia de 20 años de edad, que viajó junto con su primo:

De aquí [Saltillo, Coahuila] ya no se puede tomar el tren porque si agarra el tren de aquí hasta Reynosa nos van a fregar los de la letra ${ }^{32}$ que ya saben que uno es migrante y ya lo empiezan a extorsionas o a quitarle lo que llevan (Mario).

Sin embargo, las entrevistas no muestran cambios en ruta principal escogida, lo que puede relacionarse con lo mencionado previamente sobre la búsqueda de la ruta más corta hacia los EUA, debido a la necesidad de reducir los costos.

\section{Estrategias que involucran el acompañamiento}

Al inicio del tránsito se puede desplegar o no una estrategia que involucre acompañamiento. En principio, ir acompañado puede resultar "un recurso estratégico", por cuestión de los recursos económicos, la información y la protección que puede brindar el acompañante. Lo que podría suponer una ventaja a la hora de superar los obstáculos del camino. Así la mayoría de los entrevistados fueron acompañados durante la totalidad o parte de su recorrido hasta Saltillo. Sin embargo, las entrevistas revelaron una diversidad de situaciones por las que atraviesan que les lleva a modificar estas condiciones, y ponen en duda que ir acompañado resulte una estrategia ventajosa en todo momento.

Una de las estrategias comunes durante el tránsito es contar con acompañamiento cuando no se cuenta con recursos económicos. En casos como el de Edgar, un migrante de 25 años que sin experiencia partió

\footnotetext{
32 Banda delictiva conocida como "los de la letra" o "los zetas" que opera en gran parte del territorio noreste del país.
} 
acompañado de Honduras, pero quedó solo durante el tránsito, encontrar un nuevo acompañante se vuelve su única solución para continuar:

...me quedé solito ahí yo no quería seguir camino por motivo de que yo no conocía, pero luego yo me encontré un amigo ahí y me dijeron 'anda no te agüites cabrón, echémosle ganas' y yo les dije 'no tengo dinero, no llevo comida ni nada' les dije, 'no te agüites cabrón aquí llevamos en la mochila'... (Edgar).

Esta forma de estrategias de acompañamiento suele estar presente a lo largo del trayecto, sin embargo, los recursos económicos no son la única razón para querer contar con compañero/s para el camino, las necesidades de información también son fundamentales. Esto se presenta en casos como el de Francisco, un migrante de 23 años que viajaba solo y sin experiencia migratoria, él busca acompañamiento tras ser asaltado. Ese evento de violencia parece haberlo hecho percatarse de la necesidad de unirse a un migrante con información sobre los contratiempos a evadir durante el trayecto. En ocasiones, cuando el migrante se considera autosuficiente, la estrategia puede ser justo la contraria: ir solo. Lucio, un migrante de 19 años sin experiencia migratoria que viajaba solo y contaba con recursos económicos - pues su padre le enviaba dinero desde EUA-:

...En nadie confío, así en personas que me llegan 'esto y esto', por más tonto que se mire, por más sucio que se mire, por más inocente que se mire no se puede confiar en él... (Lucio).

Esta preferencia por viajar sin acompañamiento es compartida por otros migrantes entrevistados con experiencia, aunque por diversas razones. Ramón (28 años) ve el acompañamiento como un riesgo relacionado con el crimen organizado, por lo mismo procura alejarse de zonas concurridas por el flujo migratorio. Asimismo, Norberto da cuenta de otro riesgo de ir acompañado, en este caso relacionado con las prácticas de los agentes del Estado: “... siempre me ha gustado andar solo porque la policía luego cree que uno es coyote..." (Norberto). Esta actitud de rechazo hacia el acompañamiento parece presentarse en migrantes que cuentan con recursos y capacidades, expresadas como parte de los ejes transversales -contar con recursos económicos, con la información necesaria y ser hombre-, para realizar el tránsito sin ayuda.

Al margen de esto, aunque no hubo demasiada información sobre la relación entre las estrategias de acompañamiento y el género, de los cuatro informantes mujeres ninguna viajó sola y las que se quedaron sin acompañante durante el trayecto esperaron a encontrar a alguien más para continuar su tránsito. Como subrayaba Cristian, no es lo mismo ser hombre que mujer en el camino. Por otra parte, en diversas ocasiones informantes varones realizaron partes del trayecto sin compañía, e incluso, como se observa en al párrafo anterior, la rehúsan. 


\section{Estrategias que involucran acciones ante dificultades directas (agentes del Estado y criminales)}

.... lo mejor creyó que porque venía embarazada la iban a perdonar y lo que hicieron fue meterle un machetazo en su barriga... (Marcos)

Las estrategias aquí analizadas se desarrollan ante dos actores específicos: agentes del Estado y criminales. Los encuentros con los mismos se produjeron en diversas entidades federativas. En ambos casos, la estrategia predilecta para lidiar con ellos fue hacer uso de los recursos económicos mediante sobornos, el pago de extorsiones, cuotas por pasar, etcétera. “... Si se deja quitar el dinero uno no hay problema pásele, pero si uno no da dinero lo entregan a migración" (Rogelio y Brandon).

Sin embargo, esto se complejiza al momento de ser abordado por criminales, pues viajar con cantidades de dinero consideradas altas, 1600 pesos en uno de los casos registrados, puede tener impactos negativos. Un ejemplo de ello es el caso de Ernesto, quien tras un asalto fue secuestrado pues sus asaltantes, quienes 'querían pedir más dinero'. Esto se relaciona con que: “...si te agarran y traes mucho dinero 'hey, ese tiene familia'..." (Pablo). Las estrategias no sólo se conforman de formas diversas, sino que sus implementaciones son percibidas de formas distintas por los agresores. En muchos de los encuentros relatados la capacidad de agencia de los migrantes se vio seriamente afectada, o incluso anulada. Francisco, quien con 23 años no contaba con experiencia migratoria y viajaba solo, fue asaltado por dos personas con armas de fuego y menciona cómo ante la agresividad que presentaron les dijo:

...les pido que no me vayan a matar porque allá dejé a mi familia y no quería venir a morir tan largo pues. Me dicen 'no, nomás te vamos a robar todo lo que traes'... (Francisco).

Si bien a Francisco le roban, sale físicamente indemne, así parece haber conductas que pueden mitigar la violencia del evento criminal. Es difícil establecer que estos comportamientos son estrategias pues se presentan en eventos donde el migrante parece no tener opciones, sin embargo, dan cuenta de que sus acciones tienen un impacto real ante sus agresores. En este sentido otros migrantes dan cuenta de los comportamientos adecuados: “...uno sí es tranquilo y no les dice nada pues no le van a hacer nada, pero si se pone uno al brinco ya es diferente..." (Lucio). Toman relevancia condiciones más difíciles de dimensionar en estos eventos, relacionadas con la actitud misma del migrante y su capacidad de interactuar o generar empatía con otros individuos. En contraposición a esto puede observarse el caso destacado por Marcos, quien observó en el tren una situación donde una mujer embarazada confronta a un grupo de delincuentes: 
... y les empezó a gritar (...) así vino con palabras feas y les dijo así 'hijos de la gran puta' que 'déjenos pasar, nosotros tenemos hambre allá en nuestro país que por eso venimos para acá' y no le gustó a uno de ellos y sacó su machete y le rajó la panza así [hace una seña horizontal a lo largo del estómago], le dio un machetazo y le rajó así, la morra no más se quedó viendo así para enfrente... (Marcos).

En la mayoría de las situaciones registradas los migrantes no lograron librarse completamente de los criminales sino mitigar los impactos negativos. Otro caso es el de Alberto, quien se adelanta al cobro de los garroteros en el tren y les convence de que no lo bajen a él ni a su esposa y sobrina a pesar de no contar con la cuota:

...Yo hablé con uno de los garroteros que cuidan ahí, un muchacho, yo le hablé al puro chile, le dije 'mira, yo quiero irme pa Saltillo mira, yo sé que están bajando a la gente aquí' le digo, 'yo sé', le digo, haceme el favor, no me vayas a bajar... (Alberto).

El género supone diferencias en cuanto a la forma que los migrantes experimentan estos eventos. En ocasiones las mujeres se ven orilladas a utilizar su propio cuerpo como forma de pago para evitar otra clase de violencia o la deportación. Andrea, una migrante sin experiencia, de 28 años, que viajaba acompañada por su primo se ve obligada de este modo a entablar relaciones sexuales con sus agresores, durante un secuestro, para evitar que la expongan a las mismas situaciones que a sus compañeros: torturas, golpes y electroshocks. El uso del cuerpo como estrategia aparece también ante agentes del Estado, entre los informantes destaca su práctica ante un retén de la PGR con el fin de obtener salvoconducto.

\section{Reflexiones finales}

El trazo cuantitativo de la frontera vertical permitió observar cómo se trata de un fenómeno que involucra tanto a las acciones del Estado como a acciones de los criminales y el crimen organizado. Los migrantes hondureños entrevistados, pese a su situación precaria, dieron cuenta de una agencia considerable que materializan en estrategias para lidiar con los riesgos presentes a lo largo de la misma; sin embargo, estas no se pueden considerar una panacea para sortear los obstáculos del camino y salir indemnes por completo.

En el análisis de las estrategias preventivas permite observar que mientras los migrantes utilizan frecuentemente rodeos para evitar riesgos, no se registraron casos en que el migrante cambiase la ruta principal seguida. Lo que puede estar relacionado con la idea seguir el trayecto más corto y que involucre menores costos, aunque esto signifique correr mayores riesgos. Otro aspecto destacado en la prevención, es que tanto ir sólo como acompañado puede constituirse en 
estrategias realizadas por los migrantes hondureños; cada una de ellas cuenta con pros y contras según la situación en la que se presenten.

En cuanto a las estrategias al estar en contacto con agentes del Estado o criminales, se presenta un panorama complejo para el análisis pues la agencia de los migrantes se reduce sustancialmente. En este sentido su capacidad para desplegar estrategias exitosas se ve disminuida y los límites entre lo que es una estrategia y lo que no, parece difuminarse. Una vez que los migrantes se enfrentan cara a cara con las vicisitudes de la frontera vertical sus estrategias suelen tomar una naturaleza mitigadora de daños.

El éxito o fracaso del migrante en el despliegue de una cierta estrategia podrá impactar en sus futuras actuaciones y en las de otros migrantes con quienes comparta experiencias. Esto determina que tanto las estrategias preventivas -elección de rutas y medios de transporte y acompañamiento-, como las que se desplegaron en contacto directo con un riesgo, están interrelacionadas. Es decir, el contacto con un riesgo no sólo les lleva a implementar una estrategia sino posteriormente a generar una estrategia preventiva para evitar la repetición del suceso.

Como cabía esperar, las entrevistas apuntan a que quien cuenta con más recursos económicos e información pueden elegir rutas y medios de transporte menos riesgosos, tienen menos necesidad de ir acompañados y llegado el caso lidian con los agentes del Estado y criminales, mitigando las consecuencias negativas de tales encuentros. Ambos elementos van cambiando a lo largo del camino, si bien los recursos económicos se reducen, en cambio, la información de la que disponen los migrantes se incrementa. Por otra parte, aunque las mujeres hondureñas entrevistadas iban acompañadas, aun así reportaron sufrir graves abusos por parte de agentes del estado y criminales.

Futuras investigaciones deberán analizar aspectos más complejos de las características del migrante relacionados a su personalidad y capacidad de interacción, pues parecen tomar una gran importancia en la implementación de estrategias. Es necesario continuar analizando estas estrategias con el fin de comprender los impactos de la frontera vertical mexicana en la agencia de los migrantes, y en su capacidad no sólo para proseguir su migración sino para mantenerse con vida.

\section{Bibliografía}

ANGUIANO TÉLLEZ, María Eugenia; TREJO PEÑA, Alma Paola. Vigilancia y control en la frontera México-Estados Unidos: efectos en las rutas del flujo migratorio internacional. Papeles de Población, v. 13, n. 51, 2007, p. 45-75.

CALVA, Luis, et alii. Principales Resultados de la Encuesta Sobre Agresión y Abuso a Migrantes (EAAM) devueltos por las autoridades migratorias, 2012. México: El Colegio de la Frontera Norte, 2015. Disponible en: <http://www.colef.mx/ wp-content/uploads/2015/03/EAAM_19_marzo.pdf >. Consultado: 01.02.2016. 
CASILLAS, Rodolfo. Una vida discreta, fugaz y anónima: los centroamericanos transmigrantes en México. Mexico: SEGOB-Conacyt, 2006.

CASILLAS, Rodolfo. Los migrantes indocumentados: su vulnerabilidad y la nuestra. In ARMIJO CANTO, Natalia. Migración y seguridad: nuevo desafío en México. México: CASEDE A. C., 2011, p. 145-164.

CASTILLO, Manuel Ángel. Las políticas hacia la migración centroamericana en países de origen, de destino y de tránsito. Papeles de población, v. 6, n. 24, 2000, p.133-157.

EAAM. Encuesta sobre Agresión y Abuso a Migrantes. El Colegio de la Frontera Norte, octubre 2011-septiembre 2012.

FERNÁNDEZ CASANUEVA, Carmen. Movilidad y asentamiento: estrategias de migrantes y trabajadores transfronterizos para vivir y laborar en la región de El Soconusco, Chiapas. In SERRANO, Javier; MARTíNEZ, Germán (eds.). Una aproximación a las migraciones internacionales en la frontera sur de México. México: Colegio de la Frontera Sur, 2009, p. 53-70.

GONZÁLEZ VELÁZQUEZ, Eduardo. Frontera vertical: México frente a los migrantes centroamericanos. México: Centro Universitario UTEG, 2011.

INSTITUTO NACIONAL DE MIGRACIÓN. Boletines mensuales de Estadísticas Migratorias, 2007-2015. México: SEGOB/INM, 2007-2015.

LONG, Norman. Sociología del desarrollo: Una perspectiva centrada en el actor. México: El COLSAN y CIESAS, 2007.

PARÍS, María Dolores. Vulnerabilidad de jóvenes migrantes en el cruce indocumentado de la frontera México-Estados Unidos. Procesos mexicanos y centroamericanos. TRACE, n. 62, 2012, p. 21-35.

PROJECT COUNSELLING SERVICE. El programa frontera sur y la política migratoria en la franja. 2015. Disponible en: <www.pcslatin.org/portal/images/PFSanalisis. pdf>. Consultado: 01.02.2016.

PEDONE, Claudia; ECHEVERRI, María Margarita; GIL ARAUJO, Sandra. Entre dos orillas. Cambios en las formas de organización de las familias migrantes latinoamericanas en España en tiempos de crisis global. In ZAVALA DE COSíO, María Eugenia; ROZÉE GÓMEZ, Virginia (coords.). El género en movimiento. Familia y migraciones. México: El Colegio de México, 2014, p. 109-138.

REDODEM (Red de Documentación de las Organizaciones Defensoras de Migrantes). Migrantes Invisibles, violencia tangible. Informe 2014. 2015. Disponible en: <http://fm4pasolibre.org/pdfs/informe_migrantes\%20invisibles_ redodem2015.pdf>. Consultado: 01.02.2016.

RIVAS CASTILLO, Jaime. ¿Víctimas nada más?: migrantes centroamericanos en el Soconusco, Chiapas. Nueva Antropología, 2011, v. 24, n. 74, p. 9-38.

RUIZ, Olivia. La Migración Centroamericana en la Frontera Sur: un Perfil del Riesgo en la Migración Indocumentada Internacional. Center for U.S. Mexican Studies, 2003. Disponible en: <http://eprints.cdlib.org/uc/item/9wh8s0bk\#page->. Consultado: 01.02.2016. 
SILVA HERNÁNDEZ, Aída. Estrategias de tránsito de adolescentes centroamericanos independientes: enfrentando la frontera vertical en México. REMHU, Revista Interdisciplinar da Mobilidade Humana, n. 44, 2015, p. 99-117.

TORRE CANTALAPIEDRA, Eduardo; ANGUIANO TÉLLEZ, María Eugenia. Viviendo en las sombras: estrategias de adaptación de familias inmigrantes mexicanas en Arizona, 2007-2015. Papeles de Población, v. 22, n. 88, 2016, p. 171-207.

TORRE-CANTALAPIEDRA, Eduardo; SCHIAVON, Jorge A. Actuar o no actuar: un análisis comparativo del rol de los estados de Chiapas y Arizona en la gestión de la inmigración. Norteamérica, v. 11, n. 1, 2016, p. 159-189.

YEE QUINTERO, José Carlos. Las víctimas del Estado: Violencia hacia migrantes hondureños, perpetrada por agentes del Estados, durante su tránsito por México. México: El Colegio de la Frontera Norte, Tesis de maestría, 2016.

Recibido para publicación en 30.06.2016 Aceptado para publicación en 29.07.2016 Received for publication in June $30^{\text {th }}, 2016$ Accepted for publication in July $29^{\text {th }}, 2016$ 